\title{
Local scale invariance and strongly anisotropic equilibrium critical systems
}

\author{
Malte Henkel \\ Laboratoire de Physique des Matériaux*, Université Henri Poincaré Nancy I, B.P. 239, \\ F - 54506 Vandœuvre-lès-Nancy Cedex, France
}

(23 October 1996)

\begin{abstract}
A new set of infinitesimal transformations generalizing scale invariance for strongly anisotropic critical systems is considered. It is shown that such a generalization is possible if the anisotropy exponent $\theta=2 / N$, with $N=1,2,3 \ldots$. Differential equations for the two-point function are derived and explicitly solved for all values of $N$. Known special cases are conformal invariance $(N=2)$ and Schrödinger invariance $(N=1)$. For $N=4$ and $N=6$, the results contain as special cases the exactly known scaling forms obtained for the spin-spin correlation function in the axial next nearest neighbor spherical (ANNNS) model at its Lifshitz points of first and second order.
\end{abstract}

PACS numbers: 64.60.-i, 05.20.-y, 11.25.Hf

The notion of scale invariance is central to the present understanding of critical phenomena. Here we are interested in strongly anisotropic criticality. There are many physical examples of this, like critical dynamics and nonequilibrium dynamics, domain growth 2 magnetic systems with competing interaction 3 or particle reaction systems such as directed percolation. By definition, these systems are characterized by the condition that the critical two-point functions $\mathcal{C}$ transform under rescaling as

$$
\mathcal{C}\left(b r, b^{\theta} t\right)=b^{-2 x} \mathcal{C}(r, t)
$$

where $r, t$ label 'space' and 'time' coordinates, $x$ is a scaling dimension and $\theta=\nu_{\|} / \nu_{\perp}$ is the anisotropy exponent (in many cases, it is also referred to as the dynamic exponent $z$ ). In this letter, we confine ourselves to strongly anisotropic equilibrium systems.

Eq. (11) can be rewritten as

$$
\mathcal{C}(r, t)=t^{-2 x / \theta} \Phi\left(\frac{r^{\theta}}{t}\right)
$$

where $\Phi(u)$ is a scaling function. Some information on the form of $\Phi(u)$ is readily available. For $r=0$, one expects $\mathcal{C}(0, t) \sim t^{-2 x / \theta}$ and for $t=0$, one expects $\mathcal{C}(r, 0) \sim r^{-2 x}$. This implies $\Phi(u) \simeq \Phi_{0}$ for $u \rightarrow 0$ and $\Phi(u) \simeq \Phi_{\infty} u^{-2 x / \theta}$ for $u \rightarrow \infty$, where $\Phi_{0, \infty}$ are generically non-vanishing constants.

Is it possible to obtain more information about $\Phi(u)$ on a general basis without going back to explicit model calculations ?

Indeed, this has been affirmatively answered in two cases. First, for isotropic critical systems, that is for $\theta=1$, the extension of eq. (1) to space-dependent rescaling factors $b=b(\vec{r})$ leads to the requirement of conformal invariance of the correlation functions. 4 (We are not going to restrict ourselves to two dimensions and shall thus sidestep the extremely powerful and elegant work done in $2 D$, as initiated in Ref. 5 .) Then the critical two-point correlation function is, up to normalizationt

$$
\mathcal{C}(\vec{r})=\left\langle\phi_{1}\left(\vec{r}_{1}\right) \phi_{2}\left(\vec{r}_{2}\right)\right\rangle=\delta_{x_{1}, x_{2}}\left|\vec{r}_{1}-\vec{r}_{2}\right|^{-2 x_{1}}
$$

where $x_{1,2}$ are the scaling dimensions of the (scalar) fields $\phi_{1,2}$ which are assumed to be quasiprimary in the sense of Ref. 同.
Second, for $\theta=2$, the extension of eq. (11) to spacetime-dependent scaling $b=b\left(\vec{r}^{t}\right)$ leads to the requirement of Schrödinger invariance. 6 - Since this correspends to the 'non-relativistic' limit of the conformal group, local fields $\phi_{i}$ are characterized by two quantum numbers, the scaling dimensions $x_{i}$ and the masses $\mathcal{M}_{i} \geq 0$. For scalar quasiprimay fields, the two-point function is, up to normalization 9.10

$$
\begin{aligned}
& \left\langle\phi_{1}\left(\vec{r}_{1}, t_{1}\right) \phi_{2}^{*}\left(\vec{r}_{2}, t_{2}\right)\right\rangle=\delta_{x_{1}, x_{2}}\left(t_{1}-t_{2}\right)^{-x_{1}} . \\
& \cdot \delta_{\mathcal{M}_{1}, \mathcal{M}_{2}} \exp \left(-\frac{\mathcal{M}_{1}}{2} \frac{\left(\vec{r}_{1}-\vec{r}_{2}\right)^{2}}{t_{1}-t_{2}}\right)
\end{aligned}
$$

with $t_{1}>t_{2}$. In comparing eqns. (3, 1), we note that the first line of (4) is similar to the conformal invariance result, while the terms containing the masses reflect the non-relativistic nature of the problem for $\theta=2$. For $\theta=1$, eq. (3) is completely standard and there are quite a few statistical mechanics models with $\theta=2$ which reproduce (4), see Refs. 10,11.

What are common features of conformal and Schrödinger transformations which might serve as a basis for generalizing beyond $\theta=1,2$ ? For notational simplicity, we shall work from now on in two 'space' dimensions or one 'time' and one 'space' dimension, respectively, but the generalization to any number of dimensions is immediate. Working in (complex) light-cone coordinates $z=x+i y, \bar{z}=x-i y$, the conformal transformations are

$$
z \rightarrow z^{\prime}=\frac{\alpha z+\beta}{\gamma z+\delta} \quad ; \quad \alpha \delta-\beta \gamma=1
$$

and similarly for $\bar{z}$. The infinitesimal generators are $\ell_{n}=-z^{n+1} \partial_{z}$ and satisfy the commutation relations $\left[\ell_{n}, \ell_{m}\right]=(n-m) \ell_{n+m}$. The set $\left\{\ell_{ \pm 1}, \ell_{0}\right\}$ generates the Möbius transformations (5). The safee-time transformations of the Schrödinger group are 60

$$
t \rightarrow t^{\prime}=\frac{\alpha t+\beta}{\gamma t+\delta}, r \rightarrow r^{\prime}=\frac{r+v t+a}{\gamma t+\delta}
$$


(with $\alpha \delta-\beta \gamma=1$ ) which contains the Galilei group as a subgroup. As is well known from non-relativistic quantum mechanics, the wave function $\psi(r, t)$ transforms under a unitary projective representation $\mathcal{U}$ of the Galilei transformation 12

$$
\mathcal{U}^{-1} \psi(r, t) \mathcal{U}=\exp \left[\frac{i m}{2}\left(v^{2} t+2 v r\right)\right] \psi(r+v t, t)
$$

where $m \geq 0$ is the mass of the particle. This gives rise to the Bargmann superselection rule 120 already present in (4). If a wave function $\psi$ is characterized by the mass $m \geq 0$, its complex conjugate $\psi^{*}$ is characterized by $-m$. This correspondence between a field $\phi$ and $\phi^{*}$ is to be kept when going over to diffusive behaviour $m \rightarrow i \mathcal{M}$. An analogous statement applies to the full Schrödinger group.613 The infinitesimal generators must therefore contain mass terms and may be written in the form 10

$$
\begin{aligned}
& X_{n}=-t^{n+1} \partial_{t}-\frac{n+1}{2} t^{n} r \partial_{r}-\frac{n(n+1)}{4} \mathcal{M} t^{n-1} r^{2} \\
& Y_{m}=-t^{m+1 / 2} \partial_{r}-\left(m+\frac{1}{2}\right) \mathcal{M} t^{m-1 / 2} r \\
& M_{n}=-t^{n} \mathcal{M}
\end{aligned}
$$

and the non-vanishing commutators are

$$
\begin{gathered}
{\left[X_{n}, X_{m}\right]=(n-m) X_{n+m} \quad, \quad\left[X_{n}, Y_{m}\right]=\left(\frac{n}{2}-m\right) Y_{n+m}} \\
{\left[X_{n}, M_{m}\right]=-m M_{n+m}, \quad\left[Y_{n}, Y_{m}\right]=(n-m) M_{n+m}}
\end{gathered}
$$

The set $\left\{X_{ \pm 1}, X_{0}, Y_{ \pm 1 / 2}, M_{0}\right\}$ generates the transformations (6).

We now specify the conditions under which we shall attempt to consider an arbitrary value of the exponent $\theta$. These conditions are formulated as to remain as close as possible to the known situations of either conformal or Schrödinger invariance.

1. Since in both cases, Möbius transformations play a prominent role, we shall seek space-time-transformations which in the 'time' coordinate undergoes a Möbius transformation

$$
t \rightarrow t^{\prime}=\frac{\alpha t+\beta}{\gamma t+\delta} \quad ; \quad \alpha \delta-\beta \gamma=1
$$

2. The generator for scale transformations should read $X_{0}=-t \partial_{t}-\frac{1}{\theta} r \partial_{r}$.

3. Spatial translation invariance is required.

4. The generators should contain 'mass' terms, built in analogy to the mass terms for $\theta=2$ in ( 8 ).

5. We want to use these transformations to derive differential equations for the two-point functions. We shall require that when applied to a two-point functions, the generators will yield a finite number of independent conditions. Thus the operators applied to the two-point functions should provide a realization of a finite-dimensional Lie algebra.

We now proceed to list the consequences of the above assumptions. The generator $X_{n}, n=-1,0,1$ of the
Möbius transformations must contain the term $X_{n}=$ $-t^{n+1} \partial_{t}+\cdots$ and thus satisfy the commutation relations $\left[X_{n}, X_{m}\right]=(n-m) X_{n+m}$. In order to keep the 'conformal' structure of the transformations, we must require that these commutation relations are also satisfied by the final generators $X_{n}$. Then the explicit form of $X_{0}$ implies that up to mass terms, $X_{n}=-t^{n+1} \partial_{t}-\theta^{-1}(n+1) t^{n} r \partial_{r}$. Next, we study the action of $X_{n}$ on the space translation operator $-\partial_{r}$. We shall write $\theta=2 / N$ and define, up to mass terms, the operators $Y_{m}=-t^{N / 2+m} \partial_{r}$ with $m=-N / 2+k, k=0,1, \ldots$. The nonvanishing commutators of $X_{n}$ and $Y_{m}$ are

$$
\begin{aligned}
{\left[X_{n}, X_{m}\right] } & =(n-m) X_{n+m} \\
{\left[X_{n}, Y_{m}\right] } & =\left(N \frac{n}{2}-m\right) Y_{n+m}
\end{aligned}
$$

In particular, $\left[X_{1}, Y_{-N / 2+k}\right]=(N-k) Y_{-N / 2+k+1}$. Thus, the repeated action of $X_{1}$ on $Y_{-N / 2}=-\partial_{r}$ is creating an infinite set of generators. This can only be truncated if $N=2 / \theta$ is a positive integer, $N=1,2, \ldots$. Therefore the list of possible values of $\theta$ is

$$
\theta=\frac{2}{N}=2,1, \frac{2}{3}, \frac{1}{2}, \frac{2}{5}, \frac{1}{3}, \ldots
$$

A few remarks are in order. The 'conformal' properties of the tranformations sit in the 'time' direction. It should thus be the temporal degrees of freedom which render the system critical. Therefore one should expect that the results for the two-point function to be derived below should apply independently of whether or not the 'spatial' degrees of freedom by themselves furnish a critical system. One might think of interchanging the roles of 'space' and 'time' coordinates and thus obtain a set of anisotropy exponents $\theta=\frac{1}{2}, 1, \frac{3}{2}, 2, \ldots$ To do this, however, one must impose 'conformal' invariance on the spatial degrees of freedom and this means that the spatial degrees of freedom alone should describe a system at a critical point. While that would be fine for a study of critical dynamics, many other examples of strongly anisotropic critical systems are not at a static critical point. In $(1+1) D$, however, this distinction should not be very important, since a one-dimensional subsystem with short-ranged interactions cannot order by itself.

Finally, we have to see whether it is possible to include mass terms into the generators $X_{n}, Y_{m}$ without spoiling the commutator relations (10). Indeed, this can be done. The details of this calculation will be presented elsewhere, here we merely quote the result. One solution for the generators $X_{1}$ and $Y_{-N / 2+1}$ (which generate the so-called 'special' and 'Galilei' transformations) is

$$
\begin{aligned}
X_{1} & =-t^{2} \partial_{t}-N t r \partial_{r}-\alpha r^{2} \partial_{t}^{N-1} \\
Y_{-N / 2+1} & =-t \partial_{r}-\frac{2 \alpha}{N} r \partial_{t}^{N-1}
\end{aligned}
$$

where $\alpha$ is a dimensionful, in general non-universal, constant which parametrizes the mass term. When applying these generators to a two-point function $\mathcal{C}=\left\langle\phi_{1} \phi_{2}\right\rangle$ where the fields are characterized by two quantum numbers: the scaling dimension $x_{i}$ and the 'mass' $\alpha_{i}$, consistency can only be achieved if 


$$
\alpha_{1}=(-1)^{N} \alpha_{2}
$$

We point out that for systems with $N$ even, the distinction between $\phi$ and $\phi^{*}$ becomes unnessary. In principle, it is even possible to introduce a universal mass constant $\alpha$ which is the same for all fields. On the other hand, for $N$ odd, the $\alpha_{i}$ must be kept as peculiar quantum numbers of the fields $\phi_{i}$. To each field $\phi_{i}$, characterized by the numbers $\left(x_{i}, \alpha_{i}\right)$, there is a conjugate field $\phi_{i}^{*}$ characterized by $\left(x_{i},-\alpha_{i}\right)$. Furthermore, it can be checked using (13) that the two-particle operators built from the $X_{n}, Y_{m}$ provide on $\mathcal{C}$ a realization of the Lie algebra 10 .

Two special cases can be easily recognized. For $N=2$, we recover the familiar conformal algebra, with $X_{n}=$ $\ell_{n}+\bar{\ell}_{n}$ and $Y_{n}=i\left(\ell_{n}-\bar{\ell}_{n}\right), n=-1,0,1$, provided that the 'mass' $\alpha=-c^{-2}$ (where $c$ is the speed of light, normally set to $c=1$ when introducing light-cone coordinates $z, \bar{z}=t \pm \sqrt{\alpha} r$ ). For $N=1$, we recover the generators (8) of the Schrödinger algebra, with $\alpha_{i}=\frac{1}{2} \mathcal{M}_{i}$.

We are now ready to calculate the two-point function explicitly. If $X_{n}^{(a)}$ is the generator $X_{n}$ acting on particle $a, a=1,2$ (and similarly for the $Y_{m}$ ), the two-particle operators are $\widetilde{X}_{n}=X_{n}^{(1)}+X_{n}^{(2)}$. We are interested in the two-point function

$$
G\left(r_{1}, r_{2} ; t_{1}, t_{2}\right)=\left\langle\phi_{1}\left(r_{1}, t_{1}\right) \phi_{2}^{*}\left(r_{2}, t_{2}\right)\right\rangle
$$

and the covariance of $G$ is expressed through the conditions (meaning that the $\phi_{i}$ are quasiprimary ${ }^{5}$ )

$$
\begin{aligned}
\widetilde{X}_{0} G & =\frac{x_{1}+x_{2}}{\theta} G, \quad \widetilde{X}_{1} G=\left(\frac{x_{1}}{\theta} t_{1}+\frac{x_{2}}{\theta} t_{2}\right) G \\
\widetilde{X}_{-1} G & =\widetilde{Y}_{m} G=0
\end{aligned}
$$

with $m=-N / 2,-N / 2+1, \ldots, N / 2$. We write $t=t_{1}-t_{2}$ and $r=r_{1}-r_{2}$. In addition, we put $\zeta=\left(x_{1}+x_{2}\right) / \theta$. The scaling of the two-point function can be written as

$$
G=G(r, t)=\delta_{x_{1}, x_{2}} \delta_{\alpha_{1}, \alpha_{2}} r^{-2 x_{1}} \Omega\left(\frac{t}{r^{2 / N}}\right)
$$

where $\Omega(v)$ satisfies the differential equation

$$
\alpha_{1} \Omega^{(N-1)}(v)-v^{2} \Omega^{\prime}(v)-\zeta v \Omega(v)=0
$$

subject to the boundary conditions $\Omega(0)=$ cste. and $\Omega(v) \sim v^{-\zeta}$ as $v \rightarrow \infty$. The general solution (for $N \geq 2$ ) of equation (17) is

$$
\Omega(v)=\sum_{p=0}^{N-2} b_{p} v^{p} \mathcal{F}_{p} \quad ; \quad \mathcal{F}_{p}={ }_{2} F_{N-1}\left(\frac{\zeta+p}{N}, 1 ; 1+\frac{p}{N}, 1+\frac{p-1}{N}, \ldots, \frac{p+2}{N} ; \frac{v^{N}}{N^{N-2} \alpha_{1}}\right)
$$

where ${ }_{2} F_{N-1}$ is a generalized hypergeometric function and the $b_{p}$ are free parameters. In order to check the boundary conditions, we recall the known 14 asymptotic behaviour of the $\mathcal{F}_{p}$. The leading behaviour for $v \rightarrow \infty$ for each term is of the order $\exp \left(A(N-2) v^{N /(N-2)}\right)$, where the constant $A>0$. For $N \geq 3$ the condition

$$
\sum_{p=0}^{N-2} b_{p} \frac{\Gamma(p+1)}{\Gamma\left(\frac{p+1}{N}\right) \Gamma\left(\frac{p+\zeta}{N}\right)}\left(\frac{\alpha_{1}}{N^{2}}\right)^{p / N}=0
$$

is sufficient to cancel the entire exponential contribution. Eliminating $b_{N-2}$, the final result becomes $\Omega(v)=$ $\sum_{p=0}^{N-3} b_{p} \Omega_{p}(v)$, with $b_{0} \neq 0$. The asymptotic behaviour

$$
\Omega_{p}(v) \simeq \begin{cases}v^{p} & ; v \rightarrow 0 \\ \Omega_{\infty} v^{-\zeta} & ; v \rightarrow \infty\end{cases}
$$

is found to be in complete agreement with the requested boundary conditions, where

$$
\begin{aligned}
& \Omega_{p}(v)=v^{p} \mathcal{F}_{p}-\frac{\Gamma(p+1)}{\Gamma\left(\frac{p+1}{N}\right) \Gamma\left(\frac{p+\zeta}{N}\right)} \frac{\Gamma\left(\frac{N-1}{N}\right) \Gamma\left(1+\frac{\zeta-2}{N}\right)}{\Gamma(N-1)}\left(\frac{\alpha_{1}}{N^{2}}\right)^{(p+2-N) / N} v^{N-2} \mathcal{F}_{N-2} \\
& \Omega_{\infty}=-\left(\frac{\alpha_{1}}{N^{2}}\right)^{(\zeta+p) / N} \frac{\Gamma\left(\frac{1-\zeta}{N}\right)}{\Gamma(1-\zeta)} \frac{\Gamma(p+1)}{\Gamma\left(\frac{p+1}{N}\right)} \frac{\pi \sin \left(\frac{\pi}{N}(p+2)\right)}{\Gamma\left(\frac{p+\zeta}{N}\right) \sin \left(\frac{\pi}{N}(p+\zeta)\right) \sin \left(\frac{\pi}{N}(\zeta-2)\right)}
\end{aligned}
$$

Eq. (16) together with eqs. (18, 19) or (21) gives the solution to our question. After normalization, $N-3$ of the parameters $b_{p}$ are still arbitrary.

It remains to be seen whether there exist examples which do reproduce these predictions. Here, we shall consider the spin-spin correlator in spin systems with axial next nearest neighbor interactions. 15.3 The spin Hamiltonian is

$$
\mathcal{H}=-J \sum_{(i, j)}^{\prime} s_{i} s_{j}+\kappa J \sum_{i \|} s_{i \|} s_{i \|+1}
$$

where $s_{i}$ is a $O(n)$ vector spin and the first term $(J>$ 
$0)$ describes nearest neighbor ferromagnetic interactions while the second term $(\kappa>0)$ contains next-nearest neighbor interactions along a single axis. By definition, 15 the meeting point of the paramagnetic, ferromagnetic and incommensurable phases of the model is termed a Lifshitz point (of first order) and is known to show strongly anisotropic scaling, with correlation length exponents $\nu_{\|}=\nu_{\ell 4}, \nu_{\perp}=\nu_{\ell 2}$ measured parallel and perpendicular to the axis. The anisotropy exponent $\theta=\nu_{\|} / \nu_{\perp}=1 / 2$ independently ${ }^{5}$ of the value of $n$. This corresponds to $N=4$. The fact that $\theta=\frac{1}{2}$ stays fixed at its mean-field value may point toward the existence of a hidden symmetry which prevents its renormalization 16

In the $n \rightarrow \infty$ limit one recovers the spherical (or ANNNS3) model and the spin-spin correlation function $C\left(r_{\|}, \vec{r}_{\perp}\right)=\left\langle s_{r_{\|}, \vec{r}_{\perp}} s_{0, \overrightarrow{0}}\right\rangle$ at the Lifshitz point is exactly known in $d$ dimensions. The result ist

$$
C\left(r_{\|}, \vec{r}_{\perp}\right)=C_{0} r_{\perp}^{-\left(d-d_{*}\right)} \Psi\left(\frac{d-d_{*}}{2}, \sqrt{\frac{1}{32 c_{2}}} \frac{r_{\Perp}^{2}}{r_{\perp}}\right)
$$

where $C_{0}$ and $c_{2}$ are known (non-universal) constants, $d_{*}$ is the lower critical dimension and $\Psi(a, x)=$ $\sum_{k=0}^{\infty} \frac{(-x)^{k}}{k !} \frac{\Gamma(k / 2+a)}{\Gamma(k / 2+3 / 4)}$. On the other hand, for $N=4$ eq. (16) gives $G(r, t) \sim r^{-\zeta / 2} \Omega(v)$. As for the scaling function $\Omega(v)$, we have from (21) that for $N=4$

$$
\Omega_{0}(v)=\frac{\Gamma(3 / 4)}{\Gamma(\zeta / 4)} \Psi\left(\frac{\zeta}{4}, \frac{v^{2}}{2 \sqrt{\alpha_{1}}}\right)
$$

Thus, with the correspondence $t \leftrightarrow r_{\|}, r \leftrightarrow r_{\perp}$ and $\alpha_{1}=8 c_{2}$, the order parameter scaling function for the ANNNS model at the first order Lifshitz point is exactly reproduced for the parameter ${ }^{\text {value }} b_{1}=0$.

Higher order Lifshitz point 33 can be reached by adding further axial interaction terms in (23). Second order Lifshitz points correspond to $\theta=\frac{1}{3}$ or $N=6$. We have checked that the exactly known spin-spin correlation function for the ANNNS modell 17 does agree with the scaling form (21).

A tempting open question is whether the scaling function of the spin-spin correlator of the ANNNI model at the Lifshitz point (in $3 D$ ), which still corresponds to $N=415$ can be described in the same framework with a different value of $b_{1}$. Recently, a new asymmetric six-vertex model with a $\theta=\frac{1}{2}$ critical point has been described.18 Further examples might be provided by the superintegrable chiral $N$-state Potts model, where 19 $\nu_{\tau}=2 / N, \nu_{x}=1$ at the self-dual point or else by a nonhermitian quantum chain obtained from the asymmetric clock model, where2d $\nu_{x}=0.95(4)$ and $\nu_{\tau}=0.67(4)$. The possibility of applying the above scheme to the KPZ equation which in $(1+1) D$ has $\theta=\frac{3}{2}$, seems worth exploring.21 Finally, it appears possible to extend the present approach to yield the scaling forms for the response functions out of equilibrium (as already checked 10 in a few cases for Schrödinger invariance) and to higher $n$-point functions. This will be reported elsewhere. All in all, further explicit model results will be needed in order to gauge the merits of this or any other general approach to strongly anisotropic scaling.

In conclusion, we have examined a set of infinitesimal transformations which for $\theta=2 / N, N=1,2,3, \ldots$ generalize scale invariance. We have seen how to calculate from these the two-point functions for strongly anisotropic equilibrium critical systems. Lifshitz points in the ANNNS (spherical) model apparently provide model examples which realize these transformations.

* Unité de recherche associée au CRNS no. 155.

${ }^{1}$ A. McKane, M. Droz, J. Vannimenus and D. Wolf (Eds) Scale Invariance, Interfaces and Non-equilibrium Dynamics, NATO ASI, Vol. B344, Plenum (New York 1995).

2 A.J. Bray, Adv. Phys. 43, 357 (1994).

${ }^{3}$ W. Selke, in C. Domb and J.L. Lebowitz (Eds) Phase Transitions and Critical Phenomena, Vol. 15, Academic Press (New York 1992).

4 A.M. Polyakov, Sov. Phys. JETP Lett. 12, 381 (1970). See L. Schäfer J.Phys. A9, 377 (1975) for a full discussion how conformal invariance enters into eq. (3).

${ }^{5}$ A.A. Belavin, A.M. Polyakov and A.B. Zamolodchikov, Nucl. Phys. B241, 333 (1984).

${ }^{6}$ U. Niederer, Helv. Phys. Acta 45, 802 (1972).

${ }^{7}$ C.R. Hagen, Phys. Rev. D5, 377 (1972).

${ }^{8}$ A.O. Barut, Helv. Phys. Acta 46, 496 (1973).

${ }^{9}$ M. Henkel, Int. J. Mod. Phys. C3, 1011 (1992).

${ }^{10}$ M. Henkel, J. Stat. Phys. 75, 1023 (1994).

${ }^{11}$ M. Henkel and G.M. Schütz, Int. J. Mod. Phys. B8, 3487 (1994).

12 J.-M. Levy-Leblond, Comm. Math. Phys. 4, 157 (1967); D. Giulini, Ann. of Phys. 249, 222 (1996).

13 M. Perroud, Helv. Phys. Acta 50, 233 (1977).

${ }^{14}$ E.M. Wright, Proc. London Math. Soc. 46, 389 (1940); J. London Math. Soc. 27, 256 (1952) erratum.

${ }^{15}$ R.M. Hornreich, M. Luban and S. Shtrikman, Phys. Rev. Lett. 35, 1678 (1975); Phys. Lett. 55A, 269 (1975).

16 J.L. Cardy, priv. comm.

${ }^{17}$ L. Frachebourg and M. Henkel, Physica A195, 577 (1993).

18 G. Albertini, S.R. Dahmen and B. Wehefritz, J. Phys. A29, L369 (1996) and cond-mat/9606137.

${ }^{19}$ R.J. Baxter, J. Stat. Phys. 57, 1 (1989); G. Albertini, B.M. McCoy, J.H.H. Perk and S. Tang, Nucl. Phys. B314, 741 (1989); J.L. Cardy, Nucl. Phys. B389, 577 (1993); H. AuYang and J.H.H. Perk, J. Stat. Phys. 78, 17 (1995); G.v. Gehlen, hep-th/9606001.

20 T. Vescan, G.v. Gehlen and V. Rittenberg, J. Phys. A19, 1957 (1986).

${ }^{21}$ Comparison with the scaling function for $N=3$ would imply an interchange of time and space, as discussed above. This interchange might not be trivial, since the nonlinearity of the KPZ equation does not enter into the spatial correlations (J. Krug, priv. comm.). Work along these lines is in progress. 